\title{
Interactions between Art and Mobile Robotic System Engineering
}

\author{
Francesco Mondada ${ }^{1}$, Skye Legon ${ }^{2}$ \\ ${ }^{1}$ Autonomous Systems Laboratory (ASL-ISR-DMT) \\ ${ }^{2}$ Signal Processing Laboratory (LTS-DE) \\ Swiss Federal Institute of Technology (EPFL) \\ CH-1015 Lausanne \\ Switzerland \\ francesco.mondada@epfl.ch \\ skye. legondepfl.ch
}

\begin{abstract}
The field of mobile robotics offers a new medium for public entertainment and art. Mobile robots can move, react, and interact in the real world, generating behaviors that can be used as a new artistic medium quite different from sculptures, drawings or video. This new medium, like other technological media such as video or the Internet, requires considerable technical know-how to be exploited successfully. The successful design of a mobile robot demands a strong interdisciplinary and systems-oriented engineering process. The addition of artistic constraints adds a new dimension to the engineering problem and reinforces the need for a coherent approach to the design.

This paper illustrates this interdisciplinary approach with six examples of robotic art and entertainment projects that demonstrate the methodological issues needed for this type of work. Several aspects of the projects are discussed, including the artistic effects on the public, the sometimes problematic interaction between artists and engineers, and details of the mechanical, electronic and behavioral designs as applied to entertainment.
\end{abstract}

\section{Introduction}

Mobile robots are an increasingly popular trend in entertainment and art. International exhibitions such as Hannover 2000 have exploited this, while completely new exhibitions like RoboFesta in Japan are centered entirely on robotics. Large entertainment companies like Sony are investing heavily in robotics, while an increasing number of films and video games feature robots, or are based entirely on robots. Artists as well have discovered robotics as a new medium for their creations, seeing in robotics a means of eliciting strong reactions and emotions in the public. While films and video games admittedly do not need to actually create real robots, most of the other domains involve at least a partial implementation. It is clear that mobile robots are assuming an increasing role in our society in general, in commercial entertainment, and in art as a means of expression. But why? 
Mobile robots bring to art and entertainment the ability to elicit very strong projections in the viewer, in the sense that observers project their own interpretation of the robot's behavior onto the robot. This tendency to ascribe intelligence and motivations to the robot that it does not actually possess has been observed by researchers for many years (well known are Penny [1] in art and Braitenberg [2] in psychology), and the authors have confirmed these effects when presenting mobile robots during exhibitions, demonstrations or presentations to the public. The key aspects for generating a successful suspension of disbelief appear to be physical mobility and autonomous behavior. These two aspects are strongly linked in mobile robotics and generate in the viewer the impression of living organisms having their own intelligence. Simple mobility is sufficient to imbue life into the object, while autonomous behavior provides the illusion of intelligence. The behaviors do not need to be very complex, but simply a good mix between unpredictable and understandable.

Other techniques can generate projections in the observer, such as a close mimicry of existing living creatures through form, sound, behavior, and mimicry through anthropomorphism in general [3]. Mobility and autonomous behavior can also be considered in a sense as mimicking living organisms, but the level of mimicry remains very general. Recent efforts have pushed robotic mimicry as far as to simulate domestic animals like cats and dogs. This level of mimicry can yield very strong projections, but can also create expectations, which can have an adverse effect as the observer risks being disappointed when the mimicry does not meet up to expectations.

Most current robotic art and entertainment does not take advantage of the full potential of the field of mobile robotics. Many artists use the popular image of robots as complex technology to hide what are in reality very simple automatons. Others explore the aspect of motion, but are unable to create real behaviors. Very few artists make a real effort to integrate the science of mobile robotics in their projects. In both art and entertainment the robot must be carefully designed in order to achieve a desired projection. The design can be superficially seen as an engineering problem, but the range of competences required to solve the problem goes far beyond classical engineering domains. Mobile robotics is already an interdisciplinary domain where engineers must integrate mechanics, optics, electronics, computer science, and artificial intelligence. The added constraint of evoking specific emotions requires an entirely new set of competences, including design, aesthetics, history, and psychology. By itself, mobile robotics is a field that calls for strong teamwork between engineers from different disciplines; robotics in entertainment and art demands a far greater level of cooperation in a team composed of more disparate backgrounds to efficiently design a coherent end product.

The following article presents several examples of art and entertainment robotics where mobility and behavior are used in such as way as to best exploit the technology itself without pushing real-world mimicry too far. For each project is presented the motivation, the implementation and the design issues involved, as well as lessons learned from what worked and what didn't work quite as well. The projects have all been developed by K-Team, a robotics company based in Morges, Switzerland, manufacturers of the miniature research robot Khepera. All projects but one were 
commercial ventures, the exception involving an artistic collaboration for an international electronic art exhibition.

\section{Example 1: Khepera Advertising Display}

The train station in Lausanne provides several displays that companies can rent to advertise their products (see figure 1). The basic problem with these displays is that nobody looks at them, with the exception of the odd traveller passing time while waiting for the next train, and even then they attract but half-hearted interest. Considering the high traffic passing through the station daily, these displays offer a high potential audience, if only a means could be found to attract their attention.

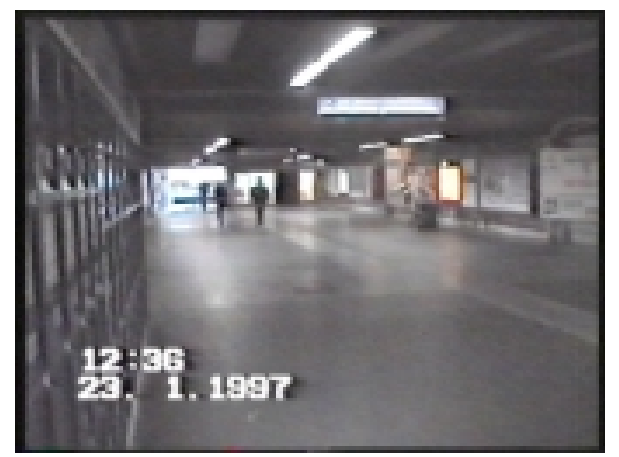

Fig. 1. Advertising displays in the Lausanne train station on the right-hand side of this underground passage

Previous experience with the Khepera robot had shown that even very simple obstacle avoidance behavior can grab the attention of quite a crowd, and so the idea of an "active" advertising display using a Khepera was developed in conjunction with local designer Krisztina Takacs-Floreano. A company in Lausanne was at this time (1997) marketing Apple's Newton PDA and purchased this idea to animate their display in the train station.

\section{Concept and Implementation}

The display consisted of a Khepera robot moving on a world map featuring a Newton PDA (see figure 2). The world and the mobility of the Khepera were used to symbolize the portability of the PDA. The Khepera robot was powered by a wire connected to a rotating contact on the ceiling, and roamed over the world map in an environment composed of several objects such as a telephone, typewriter and computer, each representing alternatives to the PDA. 


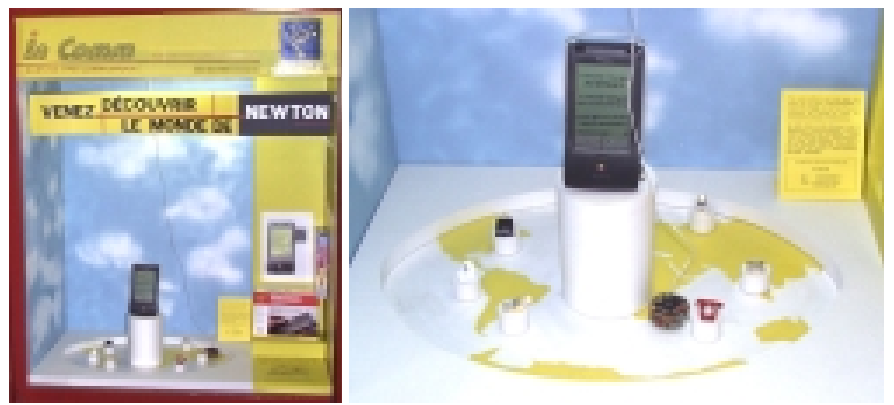

Fig. 2. The "active" display at the Lausanne train station.

Technically speaking, the robot had to simply avoid obstacles in its custom environment for roughly 20 hours per day, seven days per week. The planned duration of the display was for three months but its successful operation was extended to a full six months. The reaction to the display has been very positive and interesting. The animation attracted a large number of people, many of who passed long periods in front of the display. The display was able to catch the attention of both strolling families as well as harried businessmen (see figure 3).
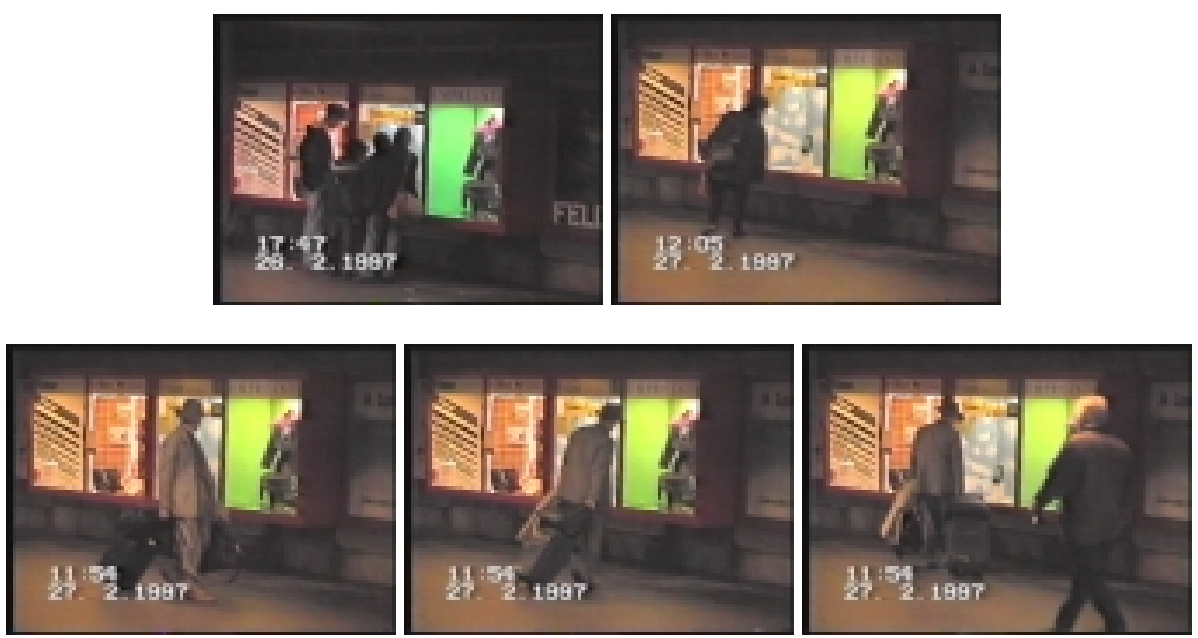

Fig. 3. A series of visitors demonstrating the efficacy of the display, with one man returning for a closer look after passing by the display

\section{Lessons Learned}

This first project demonstrated clearly how mobility and autonomous behavior can attract an audience. Mobility, especially in a display where people do not expect motion, is a strong attention-getter. Once the observer is attracted and approaches the display, the behavior begins to play a key role: the viewer studies the robot and tries 
to understand its behavior. The Khepera displayed very random behavior due to both the noisy environment seen by its optical sensors, and its navigation algorithm designed to keep the robot moving no matter what situation it found itself in. On many occasions observers would start to leave the display, but then turn back to study the robot's next movement, and then another, and another... Children especially could pass long periods fascinated by the robot, effectively trapping their parents in front of the exhibit until they were reluctantly pulled away.

From a design point of view, it was K-Team's first development that was dictated by non-technical constraints. The collaboration with the designer was very successful and the communication between both parties passed smoothly. The technical aspect, however, proved far more difficult. The behavior and environment, though apparently simple, were far more complex than imagined due to the power supply cable and the central support for the Newton. A great deal of trial-and-error was required before finding a suitable position and shape for the Newton's support such that the cable would not catch on the support and trap the robot.

\section{Example 2: Robot Avatar Dreaming with Virtual Illusions}

This second project was developed by the artist Franz Fischnaller and his group FABRICATORS in Italy, and featured the use of a Koala robot from K-Team. Fischnaller has extensive experience in exploiting new technology for artistic projects, especially works involving virtual reality [4]. He contacted K-Team for support in integrating mobile robotics into an exhibition, and K-Team joined the effort as a robotics consultant. Fischnaller merged his artistic ideas with K-Team's robotics technology for his work Robot Avatar Dreaming with Virtual Illusions, certainly the most abstract and artistic of the projects with which K-Team has been involved. It is also K-Team's only non-commercial venture of the six examples presented in this paper, though compensation came in the form of an honorary mention at Ars Electronica 99, a major international event in electronics art.

\section{Concept and Implementation}

This project combined virtual reality with real robotics, including local interaction using a joystick and remote interaction via the Internet. The robot's real-world environment was a sand arena illuminated by lateral light to emphasize the irregularity of the sand and create a suggestive and enigmatic environment, as well as to show the path of the robot (see figure 4 left). The Koala robot was modified with a transparent circular plate to generate interesting light effects, described by the authors as "a smart skin which is at the same time a catalyzer and mirror of his experience and emotions which he lives by means of the interactivity" (see figure 4 right). 

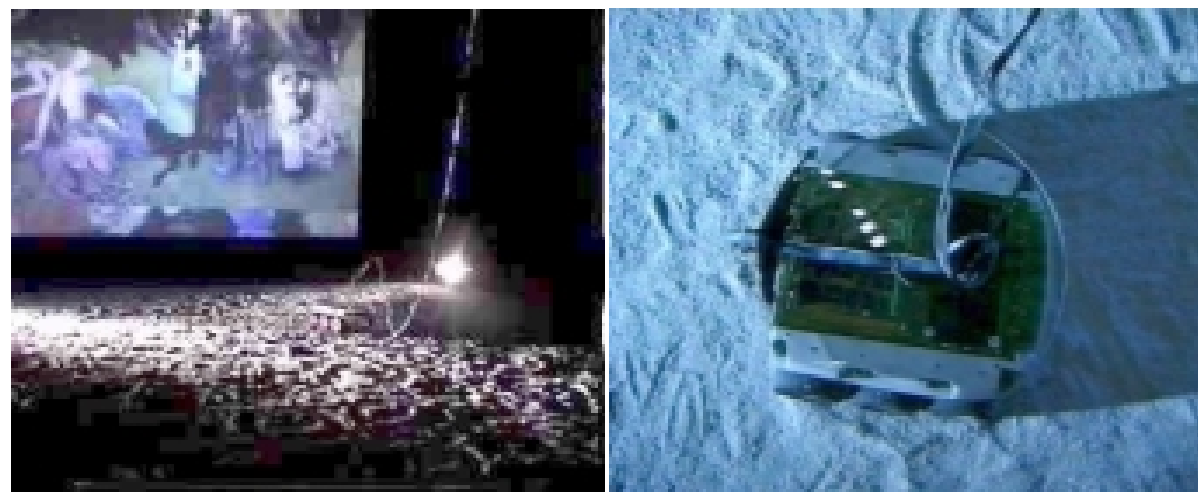

Fig. 4. The Koala in its arena

The robot had its own autonomous behaviors which were associated with the actions of the avatar in the virtual world and modified via interaction with the visitors. One interesting aspect of the association between robot and avatar is that the robot could avoid obstacles seen only by the avatar in one of several virtual worlds (shown in figure 5), resulting in enigmatic behavior to visitors only observing the real world. For more information please visit [5].
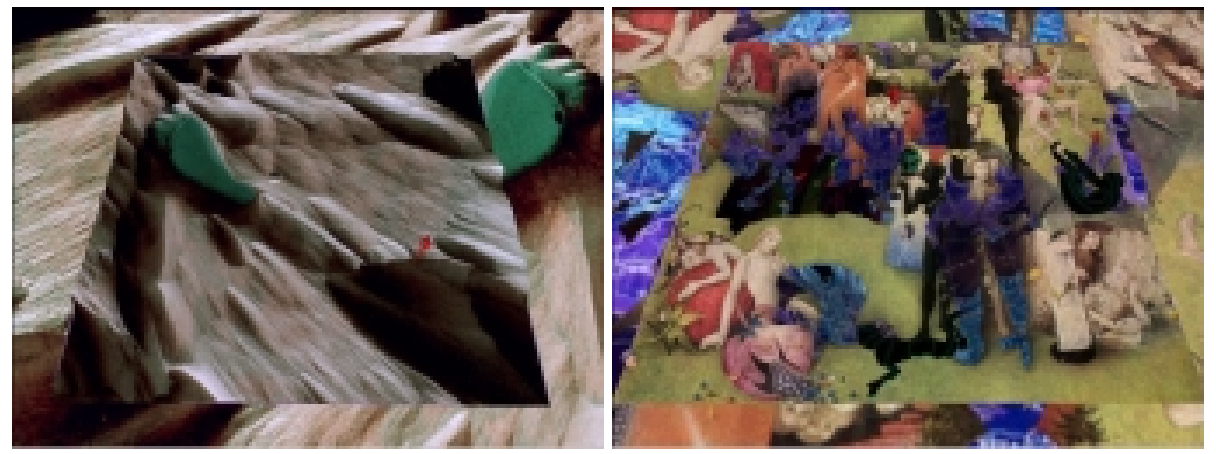

Fig. 5. Two of the worlds explored by the robot avatar

\section{Lessons Learned}

Mobility and autonomous behavior both played key roles in this work, and were also symbolic: the physical robot and its mobility are symbols for the real, visible world, while the behavior serves as a link between the physical and virtual worlds, as well as between the robot/avatar and visitor.

In the context of this paper, the most interesting aspect of this work was the nature of the interaction with Fischnaller and his group. K-Team did not perform any development for the project and remained simply a consulting partner (K-Team was more deeply involved in Fischnaller's Pinocchio project, presented as example 4). 
The robotics part of the project was actually a fairly small part of the work as a whole, but it is nonetheless significant that Fischnaller and his group programmed the robot themselves without any prior robotics experience! Although the participation of KTeam was limited to consulting, the quality of the work and the successful interaction make it a model for interdisciplinary projects. K-Team made a great effort to understand the goals and artistic constraints of the project, and propose suitable techniques to attain the desired effect. The artistic team too worked hard to understand the limits of the technology and adapt their artistic vision appropriately. Thus through a relationship of mutual respect for each other's fields was it possible to find solutions together that were both technically feasible and artistically coherent. In contrast, most partners seeking robotics technology underestimate the technical obstacles involved, and arrive with high expectations and precise demands, without any margin for adapting their concept to the limits and capabilities of mobile robots. Fischnaller fully integrated the suggestions of K-Team into his project, even the artistic ones! The end result was very successful, earning an honorary mention at the prestigious electronic art exhibition Ars Electronica 99, demonstrating the efficacy of this design methodology.

\section{Example 3: Ball Sorting Demonstration}

This purely commercial project was developed for a design firm charged with creating a robotics exhibit for an expo organized by their client, a large multi-national automotive firm. The designers approached K-Team for support in creating an attractive demonstration, and together a concept was developed whereby three Khepera robots would cooperate to "harvest" colored balls in a small arena.

\section{Concept and Implementation}

A circular arena roughly two meters in diameter was divided with painted lines into three equal wedges, each the territory of one of the three robots (see figure 6). Balls of three different sizes corresponding to each robot were introduced into the arena from the center and would end up randomly in one of the three areas. The robots roamed their terrain searching for balls, and when found would grab the ball with a gripper device. Noting the size of the ball, the robot would decide if it belonged to him, and if so then hunt for his "nest" and deposit the ball in a hole. If the ball belonged to one of his friends, he would roll over to the appropriate border between their two territories and fling the ball towards his friend, who would find the ball on his own in due course. In this roundabout way the three robots could quite quickly clear the arena of a large number of balls (see figure 7). 


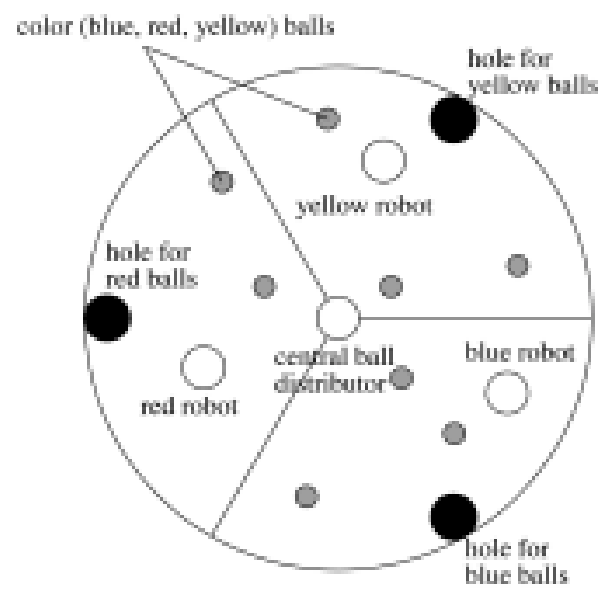

Fig. 6. The layout of the arena
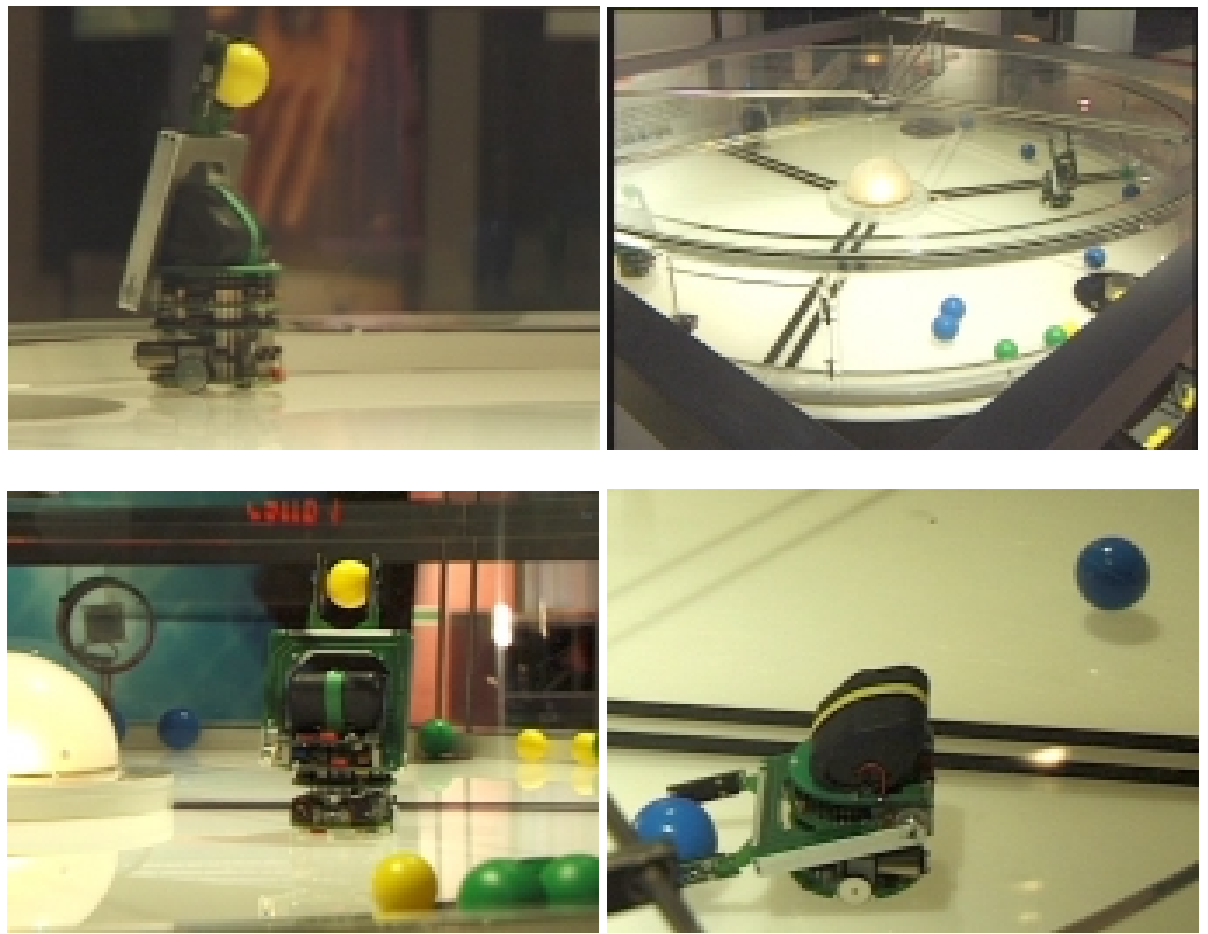

Fig. 7. Robots working together to harvest colored balls 


\section{Lessons Learned}

Here too mobility and autonomous behavior played key roles: the mobility attracted visitors to the exhibit, whereupon they remained rooted to the spot, fascinated by the robots' behavior and trying to deduce their modus operandi. The goal of the designers was thus achieved with success, and the exhibit became one of the main attractions of the entire exhibition.

This success was achieved, however, despite an often-difficult interaction with the design team. From the start of the project they rigidly defined the purpose of the installation and a list of inviolable constraints. By means of example, one such constraint was that the robots must be completely wireless, as a cable was seen to undermine the concept of computational and behavioral autonomy. This caused large difficulties, as the robots were required to run continuously 8-12 hours a day, and battery power supply remains a thorny issue in mobile robotics. After significant development the project was in its final tests just before the start of the exhibition, and to aid with their debugging the robots were connected to a computer via a cable so that the robots could display their "thought processes" on the screen. An important representative from the automotive firm happened to pass by and was fascinated by the information displayed on the screen, as it allowed him to see how the robot worked. He asked why this information wasn't part of the exhibit, and was politely told that it was difficult to transmit this information without a cable. His response was maddening: "Why not use a cable, then?" This anecdote demonstrates how a topdown design approach with no consideration for low-level technical constraints can result in absurd situations and inefficient designs.

\section{Example 4: Pinocchio Interactive}

Pinocchio Interactive is another project created by Franz Fischnaller and FABRICATORS integrating robotics, virtual reality and user interaction. It allows the visitor to explore a modern version of the classical story of Pinocchio by using a joystick to control a real robotic Pinocchio puppet or its virtual counterpart.

\section{Concept and Implementation}

The $1.8 \mathrm{~m}$ tall Pinocchio puppet is suspended, like a real puppet, by wires that control its movements. It hangs before a $3 \times 3 \mathrm{~m}$ screen showing its virtual alter ego and the virtual story (see figures 8-10). The real and virtual Pinocchio puppets can interact with each other, and can be manipulated as well by the visitors using a joystick. More information can be found at [6]. 


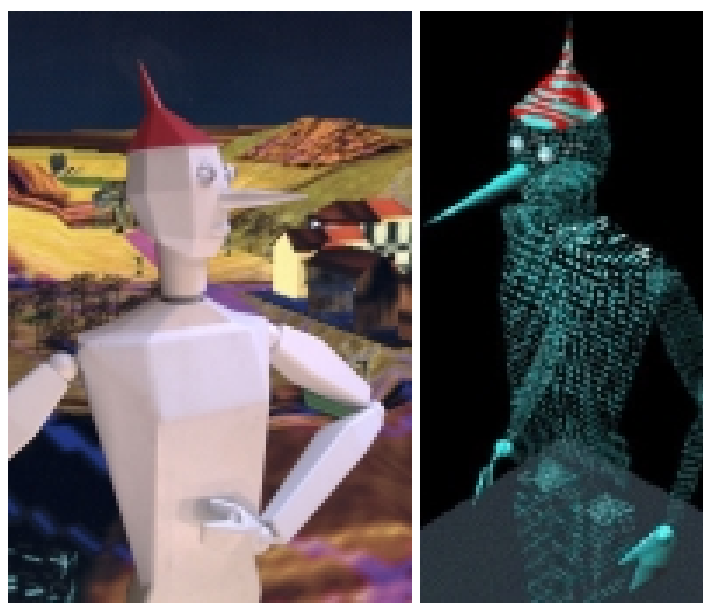

Fig. 8. The real Pinocchio puppet and its alter ego in the virtual world

The first Pinocchio puppet (still in the prototype phase) is controlled by five motors, each motor controlling one or more cables connected to its arms and legs, and is thus animated in the same manner as a classical puppet.
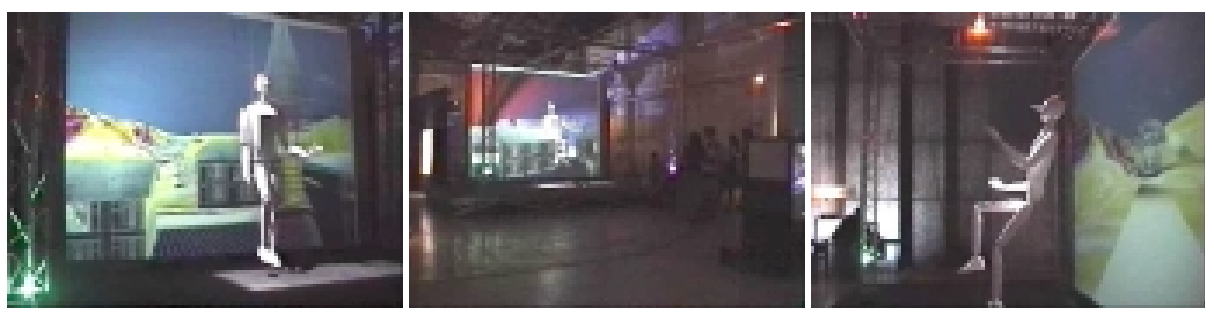

Fig. 9. The Pinocchio puppet placed in front of the screen showing the virtual world

\section{Lessons Learned}

Mobility and behavior are again present in this work, drawing the visitor into the story using a variety of actions and animations of the puppet interacting with its virtual alter ego.

Aside from difficulties in finding financing for this type of project, this experience again was a highly positive one for the same reasons as example 2, and serves as a demonstration for optimal interaction between engineers and artists. 

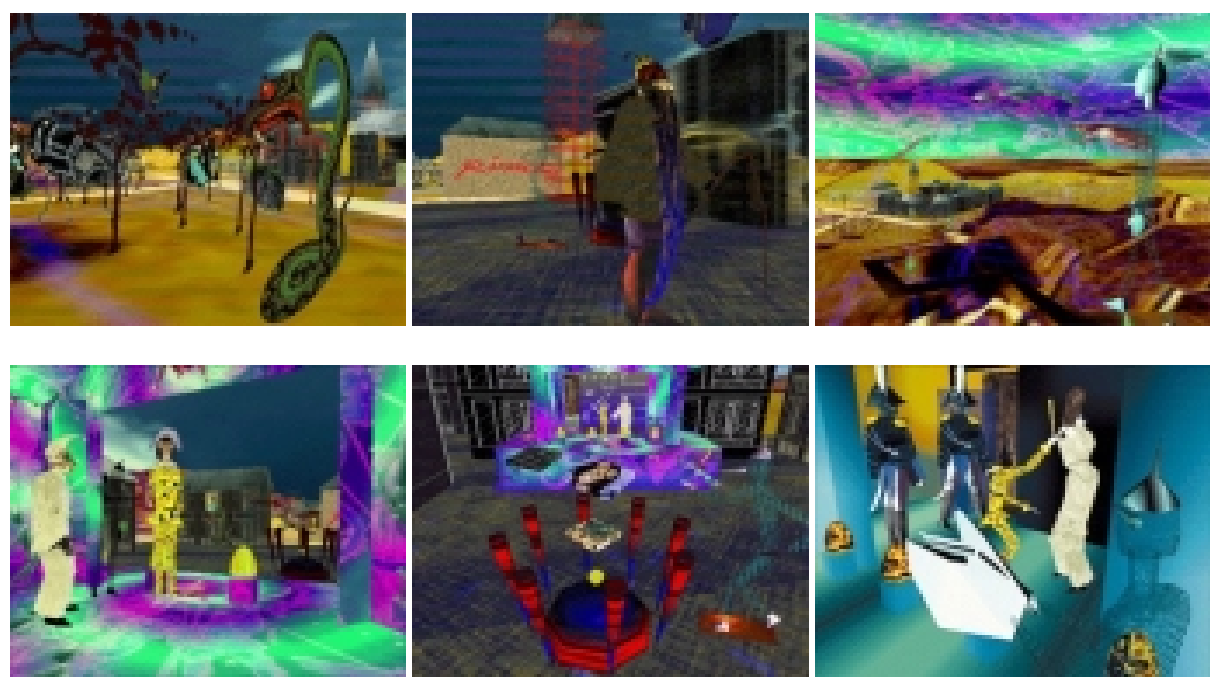

Fig. 10. Images from the virtual Pinocchio story

\section{Example 5: Flower Pot}

This project is a permanent animated sculpture located on the roof of a building at a technical school in Neuchâtel in Switzerland. As part of a renovation project the school consecrated a part of their budget to "beautify" an unsightly low gravel-roofed building in the middle of their campus. The winners of the design competition were a group of local artists, the "gruppo GPM" of Pierre Gattoni, Emmanuel Du Pasquier and Yvo Mariotti, who made contact with K-Team for aid with the implementation of the robotics of their animated sculpture.

\section{Concept and Implementation}

The animated sculpture is in reality an enormous autonomous flowerpot with the same proportions as a normal flowerpot, but with a diameter of 3 meters and weighing several hundred kilograms. In place of flowers the pot supports a 25 meter high flexible mast, designed to sway artistically in the breeze (see figure 11). The upper surface of the pot is covered with solar panels to collect energy for the internal batteries, which in turn power two large drive motors while six castor wheels ensure the stability of the system. The base of the pot is ringed with several infrared distance sensors that enable the pot to avoid obstacles, and inside the pot is hidden a reservoir and a pump to collect rainwater and to occasionally shoot plumes of water into the air. 


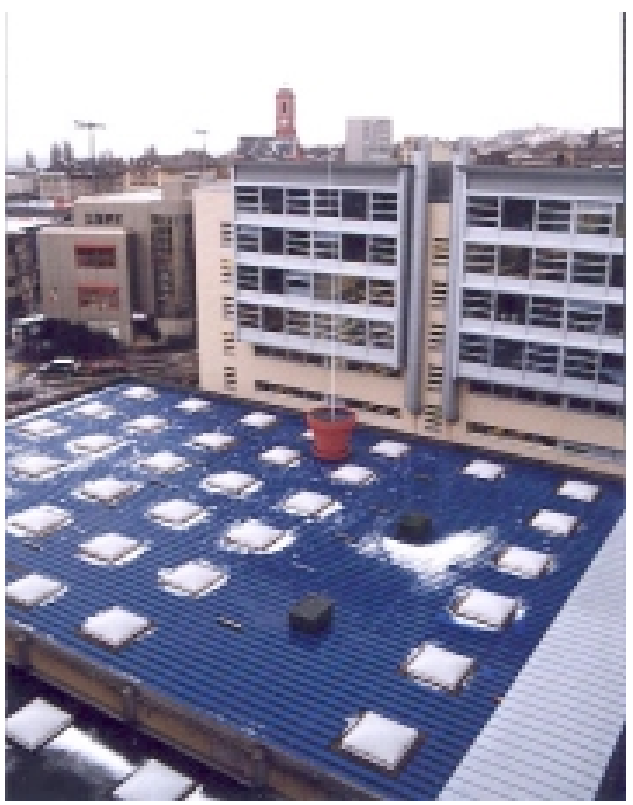

Fig. 11. The flowerpot on the roof amid the snows of winter

The flowerpot navigates around the roof avoiding obstacles (skylights and the external walls) several hours per day. An important characteristic of the system is its rather modest speed of 2 to 5 meters per hour. This creates an interesting effect: when looking at the pot it seems immobile, but each time you look it is in a different location (see figure 12). The artists draw an analogy with the moon: you do not see it move, but you always have to look around to find it again.

From a technical standpoint, the robot behaves like a (very) large Khepera robot, and is controlled by K-Team's Kameleon board with a custom extension to read the infrared sensors and control the motors. Developing a robust classical navigation algorithm with such simple sensors proved impossible, and so a Braitenberg-inspired neural network was used, with sensor parameters and positioning evolved using a genetic algorithm. The custom genetic algorithm was developed using the simulation software SysQuake (see figure 13). 

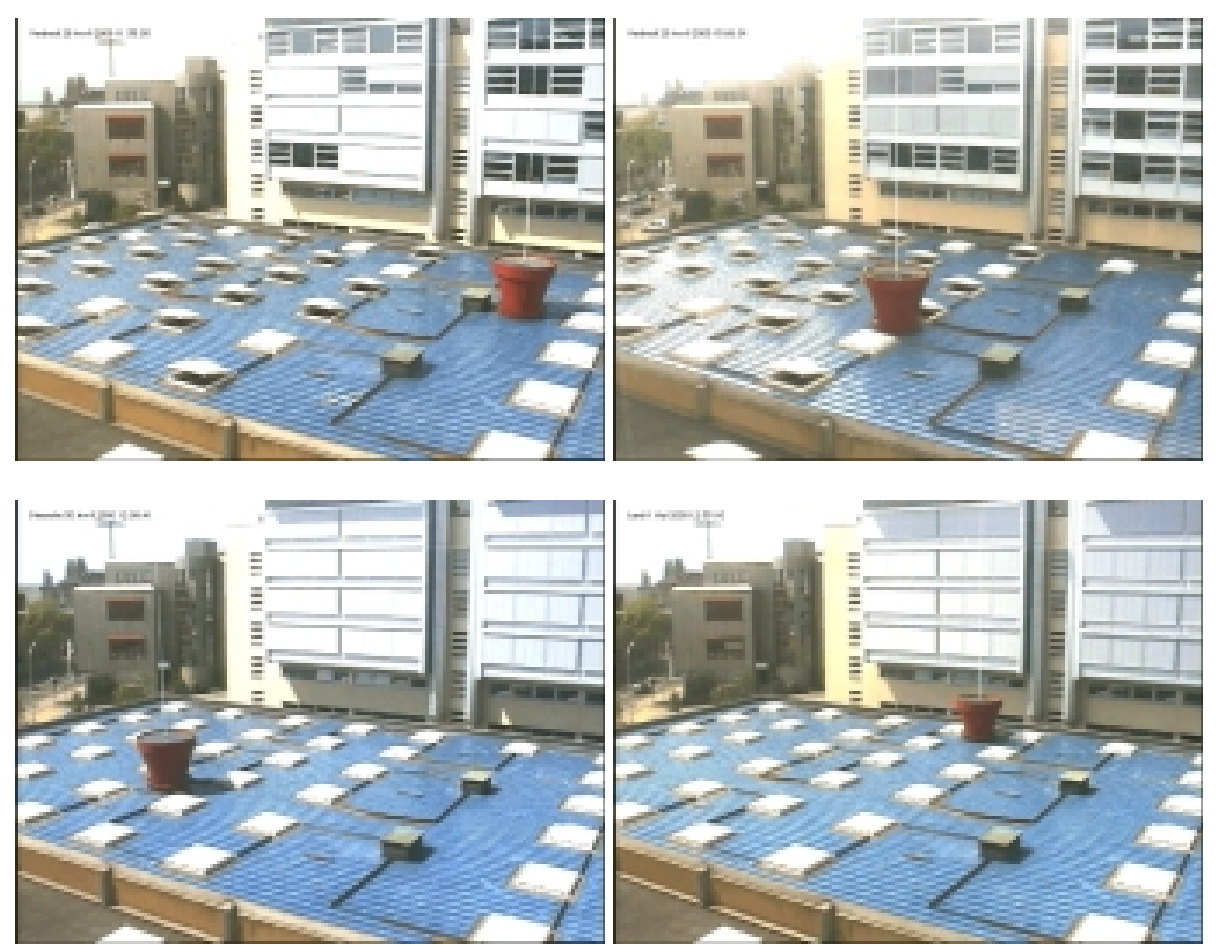

Fig. 12. The flowerpot moving around the roof over a period of 24 hours

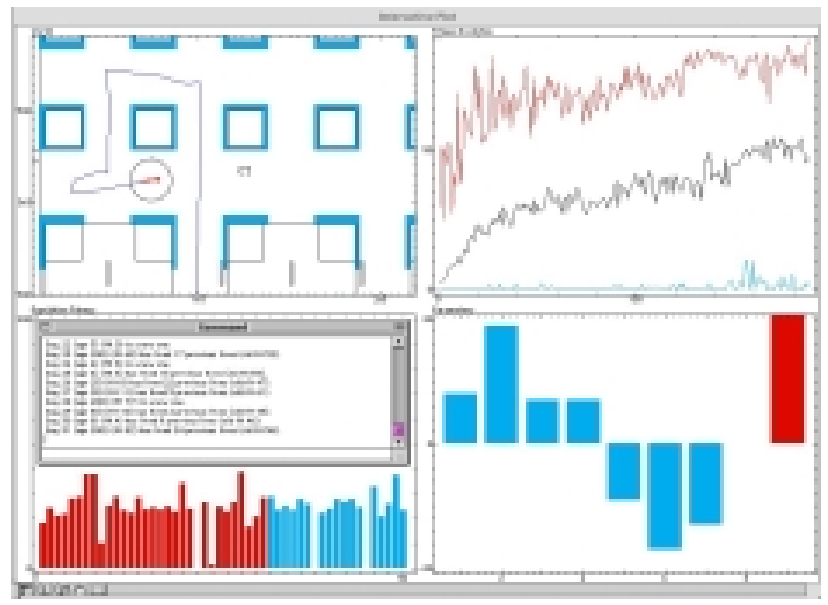

Fig. 13. Calculation of robot parameters using genetic algorithms and the mathematical simulation software SysQuake. The top-left of the image shows a map of part of the roof and the simulated trajectory of the robot 


\section{Lessons Learned}

As with the other projects, mobility and autonomous behavior are key elements, but differ from the others, however, in that these two attributes are partially hidden, or made enigmatic, by the temporal scaling of the behavior.

As K-Team was contacted after the project was defined, the interaction with the artists was limited to modification of minor details, prohibiting proper bottom-up development. In addition, as the project progressed, structural considerations due to the size of the mast required an increasingly larger and heavier pot, greatly reducing the free space between the pot and obstacles, and thus rendering the navigation problem exceedingly difficult. Interdisciplinary interaction for the development of the installation was clearly insufficient, yielding a seemingly never-ending series of technical hurdles.

\section{Example 6: Robot Theater}

This project is the most complex development in entertainment robotics undertaken by K-Team. It is a robot theatre, consisting of four robots playing the roles of mother, father, son and daughter. The project was developed for a Swiss exhibition called "Little Children: Joy and Exasperation" organized by Ethno-Expo to run from the year 2000 through 2003. The family of robots acts out a series of short scenes of domestic life, some finishing happily, and others not so happily, with the goal of educating young children and their families on the joys and frustrations of family life.

\section{Concept and Implementation}

The custom-designed robots have a cylindrical body with two hidden drive wheels, simple arms, a motorized mouth, two LED-matrix eyes and one "heart" on the body, and an internal loudspeaker (see figure 14). 


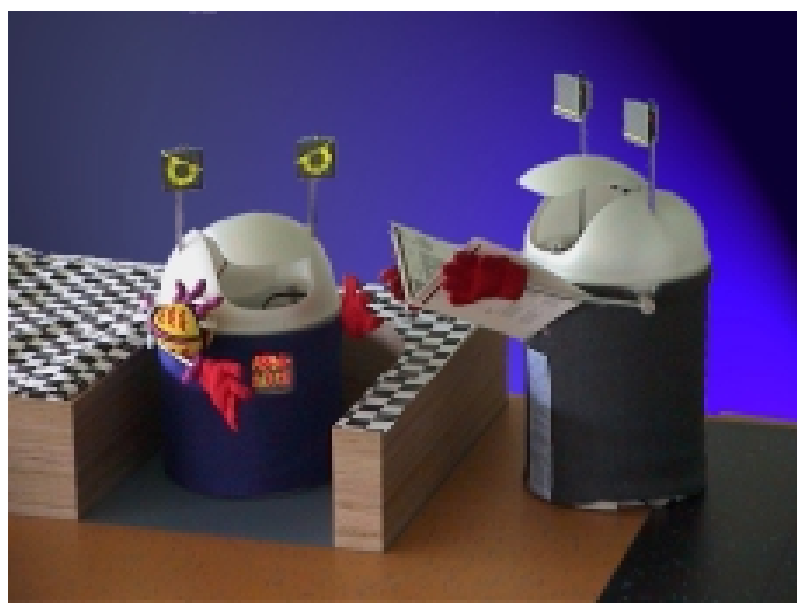

Fig. 14. Robinette and her father, two of the four robots in the family

The robots act out the scenes on a stage representing an apartment with kitchen, living room, children's bedroom, and parents' bedroom (see figure 15). The "front door" of the apartment in the kitchen leads to a small closet space where the robots rest when not onstage and recharge their batteries.
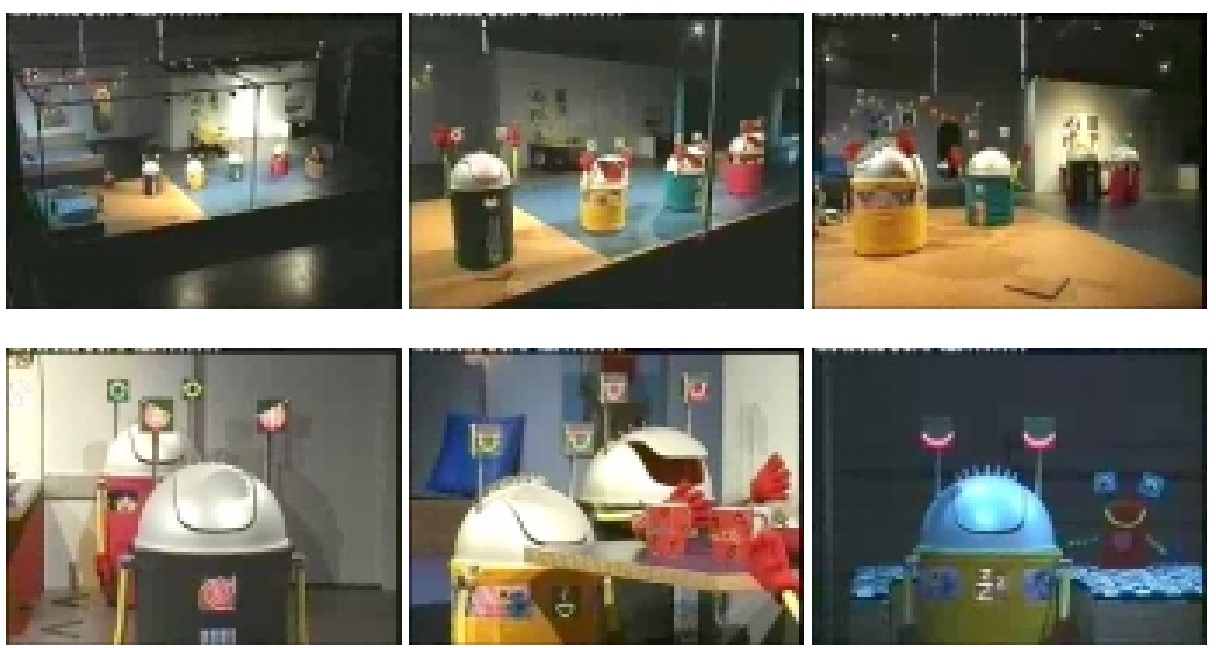

Fig. 15. Some scenes from the robot performance

The entire system is fully automatic: a central computer controls the stage accessories (television, stove, telephone, door, rotating table, the sound and lights) and controls each robot via a radio data link for movements and a radio sound channel for the voice. Each robot is constructed around a Kameleon controller board, which communicates with the host computer via a radio modem, and controls four motors (two wheels and two arms), the mouth servomotor, the LED-matrix displays, and several contact-switch sensors (see figure 16). 


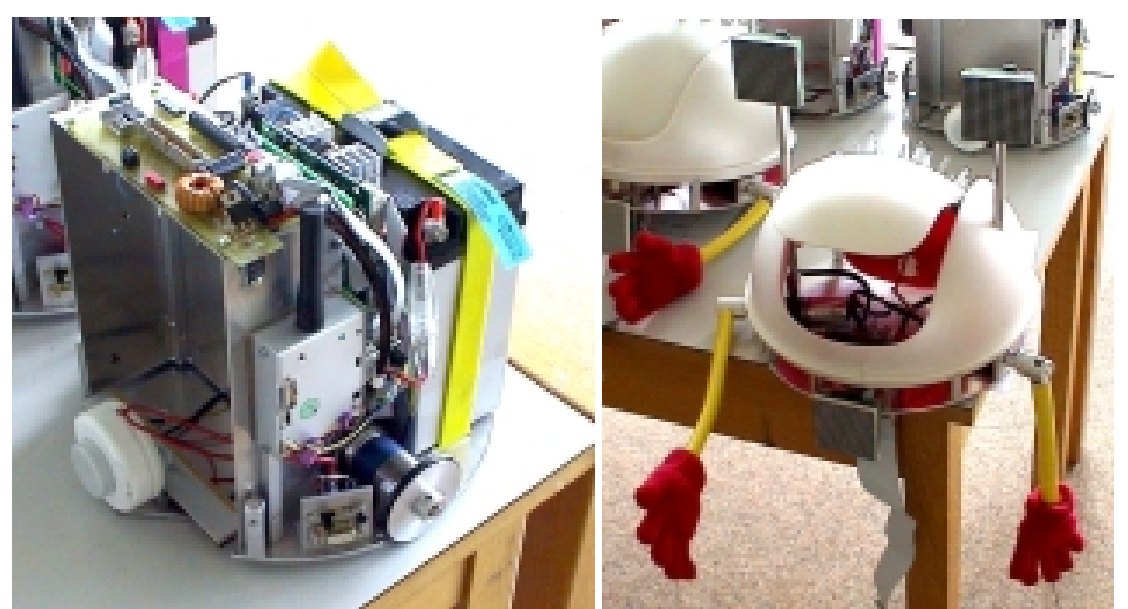

Fig. 16. Robot chassis with body removed and the head and arms of the robot.

Challenging aspects of the project included the positioning of the robots and the scripting of the scenes. Robot positioning was performed using internal odometry with regular recalibrations using mechanical landmarks, which were carefully integrated into the script and did not disturb the spectators' appreciation of the presentation. The scripting of the scenes was a complex task, and a custom multitasking event-based scripting language was developed for this purpose. Scripts were sent over the radio link to the robots and compiled on the fly to permit the coordination of the robot's complex actions, involving physical displacement, moving its arms and mouth, and controlling the LED-matrix displays. Equally importantly, the system permitted the coordination of actions between several robots, resulting in fully synchronous collective behaviors.

\section{Lessons Learned}

Unlike the other projects, the robots in this project were not behaviorally autonomous, but this was compensated for by the complexity of the scripted actions. The mobility and expressions of the robots are by far the richest of the examples presented here, and evoke strong projections of family life in both parents and children. However, as explained in the introduction, this mimicry is a double-edged sword, and the successful representation of family life also creates expectations on the part of the viewers. For example, the relatively slow speed of the robots can disappoint visitors, who project human speeds onto the robots, despite the obvious physical dissimilarity.

The design process involved a rich interaction between the artistic and technical teams, necessitated by the size and ambitious nature of the project. The interaction was facilitated by Swiss writer and researcher Jean-Bernard Billeter, whose background in both theatre and robotics aided in bridging the gap between the two teams. Aside from writing the scripts for the project, his efforts to explain technical 
constraints to the artistic team (and to sensitize a team of engineers to subtle artistic considerations) did much to smooth development.

This ambitious project demanded from the outset special efforts to link the artistic conception with the engineering design. The degree of cooperation required was at first greatly underestimated and only became clear once significant problems arose. A notable example is that of the scripting language: K-Team had planned to provide a simple linear time-based scripting language, but as development progressed it became evident that it would be wholly insufficient for coordinating a complex series of simultaneous actions of variable duration both on the robot itself, and for the scene as a whole. It was soon realized that anything less than a completely general multiagent, event-based, multi-tasking scripting language would seriously limit the artistic possibilities. This necessitated an unforeseen and costly development that underlined the need to properly define requirements and coordinate design efforts from the bottom-up.

\section{Conclusion}

The six examples presented in this article demonstrate two important aspects of robotic design for art and entertainment: the use of mobility and behavior to generate projections in the viewer, and the difficulties of coordinating artistic and technical teams to achieve this goal.

The two key elements in successfully generating emotional projections in the observer are motion and autonomous behavior. Motion is important for imbuing the robot with the illusion of life, while autonomous behavior allows the viewer to project needs, wants, and motivations onto the robot, thus bestowing it with a perceived intelligence. These projections can be used to simply entertain the visitor (as in examples 1 and 4) or if placed in a specific context (as in examples 2, 3,5 and 6) can be employed to convey a message.

The nature of the interaction between the artistic/design team and the robotics/engineering team is critical for the success of this type of development. Careful thought must be given to the design process, in particular the coordination of a joint bottom-up development between the two teams. Classical top-down clientprovider relationships between artists and engineers can result in an inefficient design process and an unsuccessful end product. Engineers must be prepared for the unrealistic expectations of non-specialists, while artists must accept that mobile robotics remains a specialized field that cannot be easily adapted to suit every need. The success of the project demands that both parties strive to understand the other's constraints and are prepared to collaborate in developing a mutually satisfactory design. 


\section{References:}

[1] S. Penny, "Embodied Cultural Agents: at the intersection of Art, Robotics and Cognitive Science", AAAI Socially Intelligent Agents Symposium, MIT, November 810,1997

[2] V. Braitenberg, Vehicles: Experiments in Synthetic Psychology. Cambridge, MIT Press, 1984

[3] S. Penny, "Why do we want our machines to seem alive?", Scientific American, 150th anniversary issue, september 1995

[4] http://www. fabricat.com

[5] http://www.fabricat.com/ROBO_HTM/robot.html

[6] http://www.fabricat.com/pinoc_home.html 